\title{
3-D acoustic shape sensitivity analysis using the fast multipole boundary element method
}

\author{
C. J. Zheng ${ }^{1,2}$, T. Matsumoto ${ }^{1}$, T. Takahashi ${ }^{1} \&$ H. B. Chen $^{2}$ \\ ${ }^{1}$ Nagoya University, Japan \\ ${ }^{2}$ University of Science and Technology of China, China
}

\begin{abstract}
The fast multipole boundary element method (FMBEM), based on the BurtonMiller formulation for 3-D acoustic sensitivity analysis, is presented in this paper in order to overcome the difficulties in the shape sensitivity analyses using the boundary element method based on the direct differentiation method.

The Burton-Miller formulation, which is a linear combination of the conventional boundary integral equation (CBIE) and its normal derivative (NDBIE), is applied to circumvent the difficulty caused by the so-called fictitious eigenfrequencies. The fast multipole method (FMM) is also employed to improve the overall computational efficiency. The sensitivity boundary integral equations of hypersingular type are obtained by the direct differentiation method. The correctness and validity of the method are demonstrated through some numerical results, from which the effectiveness of the present method is shown for 3-D acoustic shape sensitivity analyses.

Keywords: acoustics, shape sensitivity, direct differentiation method, fictitious eigenfrequency, Burton-Miller's method, fast multipole boundary element method.
\end{abstract}

\section{Introduction}

The process of computing gradients of the defined acoustic performance function associated with a structure with respect to design variables has been termed as the acoustic sensitivity analysis. Although the BEM has the incomparable superiority, which makes it widely used in the acoustic sensitivity analysis, it also has some difficulties in solving exterior acoustic problems. The first one is that the BEM with the CBIE fails to yield unique solutions for exterior problems at the eigenfrequencies of the associated interior problems. These eigenfrequencies 
are called fictitious eigenfrequencies because they do not have any physical significance. In order to tackle this difficulty, the Burton-Miller method is a more sound and effective method than any other existing ones [1].

The second difficulty, which also restricts the usage of the BEM in large-scale engineering problems, is the high computational complexity. For a problem with $N$ unknowns, a direct solver, such as the Gauss elimination method, requires $O\left(N^{3}\right)$ solution cost and storage of $O\left(N^{2}\right)$. Even worse, in the sensitivity analysis by the direct differentiation method, more integral evaluations for each pair of boundary elements are needed. However, the computation can be performed in $O(N)$ operations and $O(N)$ memory requirement by using the FMBEM, for example, in potential problems. The FMBEM was first proposed by Rokhlin [2], and a comprehensive review can be found in [3].

In this paper, the FMBEM based on the Burton-Miller formulation for 3-D acoustic problems is adopted to make the acoustic shape sensitivity analysis more efficient. The well-known hypersingular nature of the NDBIE can be evaluated without any difficulty by using the constant triangular element discretization, which also makes the FMBEM more efficient than the regularization technique because multipole expansion formulas and other translation formulas only have to be implemented for the fundamental solution and its derivatives of the Helmholtz equation.

\section{Formulations}

\subsection{Conventional boundary element method (CBEM) formulations}

The propagation of time-harmonic acoustic waves in a homogeneous and isotropic acoustic medium is described by the following Helmholtz equation

$$
\nabla^{2} p(x)+k^{2} p(x)=0,
$$

where $p(x)$ is the sound pressure at point $x$, and $k=\omega / c$ the wave number, $\omega$ the circular frequency, $c$ the acoustic velocity.

The boundary conditions for Helmholtz equation are written as

$$
\begin{gathered}
p(x)=\bar{p}(x), \quad \text { on } \Gamma_{p}, \\
q(x)=\frac{\partial p}{\partial n}(x)=i \rho \omega \bar{v}(x), \quad \text { on } \Gamma_{q}, \\
p(x)=z v(x), \quad \text { on } \Gamma_{z},
\end{gathered}
$$

where $n(x)$ denotes the unite outward normal vector at point $x, i$ the imaginary unit, $\rho$ the medium density, $v(x)$ the particle velocity and $z$ the acoustic impedance. The quantities denoted with bars are assumed to be given values on the boundary. In the case of acoustic radiation, $p(x)$ must also satisfy the Sommerfeld's radiation condition. 


\subsubsection{CBEM formulations for acoustic state analysis}

The integral representation of the solution to the Helmholtz equation is

$$
p(x)+\int_{\Gamma} q^{*}(x, y) p(y) \mathrm{d} \Gamma(y)=\int_{\Gamma} p^{*}(x, y) q(y) \mathrm{d} \Gamma(y) .
$$

The CBIE and NDBIE of the Helmholtz equation are written, as follows:

$$
\begin{aligned}
& C(x) p(x)+f_{\Gamma} q^{*}(x, y) p(y) \mathrm{d} \Gamma(y)=\int_{\Gamma} p^{*}(x, y) q(y) \mathrm{d} \Gamma(y), \\
& C(x) q(x)+f_{\Gamma} \tilde{q}^{*}(x, y) p(y) \mathrm{d} \Gamma(y)=f_{\Gamma} \tilde{p}^{*}(x, y) q(y) \mathrm{d} \Gamma(y),
\end{aligned}
$$

where $(\tilde{)}) \partial() / \partial n(x)$, the constant $C(x)$ is $1 / 2$ if $\Gamma$ is smooth around $x$, and $f$ and $f$ denotes the integral is evaluated in the sense of Cauchy's principal value and finite part of divergent integral, respectively. $p^{*}(x, y)$ is the fundamental solution, for 3-D problems it is given as

$$
p^{*}(x, y)=\frac{e^{i k r}}{4 \pi r},
$$

where $r=|x-y|$, and $q^{*}(x, y)$ is the normal derivative of $p^{*}(x, y)$.

Both eqns (6) and (7) can be used to calculate the unknown boundary state values. For exterior acoustic problems, by using either of them solely, unique solutions cannot be obtained at the fictitious eigenfrequencies. However, Burton and Miller [1] have shown that we can obtain unique solutions for all such frequencies by using a linear combination of eqns (6) and (7) in the form:

$$
\begin{aligned}
& C(x) p(x)+f_{\Gamma} q^{*}(x, y) p(y) \mathrm{d} \Gamma(y)+\alpha f_{\Gamma} \tilde{q}^{*}(x, y) p(y) \mathrm{d} \Gamma(y) \\
& =-\alpha C(x) q(x)+\int_{\Gamma} p^{*}(x, y) q(y) \mathrm{d} \Gamma(y)+\alpha f_{\Gamma} \tilde{p}^{*}(x, y) q(y) \mathrm{d} \Gamma(y),
\end{aligned}
$$

where $\alpha$ is a coupling constant that can be chosen as $i / k$ [4].

Discretizing eqn (9), collecting the equations for all the collocation points and expressing them in matrix forms result in the following form of linear algebraic equations:

$$
[H]\{p\}=[G]\{q\} .
$$

Rearranging eqn (10) gives the following system of linear equations

$$
[A]\{x\}=[B]\{y\},
$$

where $[A]$ is the system matrix, $\{x\}$ and $\{y\}$ are the unknown and known vectors.

Eqn (11) can now be solved and all the boundary state values are then known. After that, it is possible to calculate $p(x)$ at any point $x$ within the problem domain by using eqn (5). 


\subsubsection{CBEM formulations for acoustic sensitivity analysis}

In order to obtain the formulations for sensitivity analysis, eqns (5), (6) and (7) are differentiated with respect to an arbitrary shape design variable to yield

$$
\begin{aligned}
& \dot{p}(x)+\int_{\Gamma} q^{*}(x, y) \dot{p}(y) \mathrm{d} \Gamma(y)+\int_{\Gamma} \dot{q}^{*}(x, y) p(y) \mathrm{d} \Gamma(y) \\
& +\int_{\Gamma} q^{*}(x, y) p(y) \mathrm{d} \Gamma(y)=\int_{\Gamma} p^{*}(x, y) \dot{q}(y) \mathrm{d} \Gamma(y) \\
& +\int_{\Gamma} \dot{p}^{*}(x, y) q(y) \mathrm{d} \Gamma(y)+\int_{\Gamma} p^{*}(x, y) q(y) \mathrm{d} \Gamma(y), \\
& C(x) \dot{p}(x)+f_{\Gamma} q^{*}(x, y) \dot{p}(y) \mathrm{d} \Gamma(y)+f_{\Gamma} \dot{q}^{*}(x, y) p(y) \mathrm{d} \Gamma(y) \\
& +f_{\Gamma} q^{*}(x, y) p(y) \mathrm{d} \Gamma(y)=\int_{\Gamma} p^{*}(x, y) \dot{q}(y) \mathrm{d} \Gamma(y) \\
& +\int_{\Gamma} \dot{p}^{*}(x, y) q(y) \mathrm{d} \Gamma(y)+\int_{\Gamma} p^{*}(x, y) q(y) \mathrm{d} \Gamma(y), \\
& C(x) \dot{q}(x)+f_{\Gamma} \tilde{q}^{*}(x, y) \dot{p}(y) \mathrm{d} \Gamma(y)+f_{\Gamma} \dot{\tilde{q}}^{*}(x, y) p(y) \mathrm{d} \Gamma(y) \\
& +f_{\Gamma} \tilde{q}^{*}(x, y) p(y) \mathrm{d} \Gamma(y)=f_{\Gamma} \tilde{p}^{*}(x, y) \dot{q}(y) \mathrm{d} \Gamma(y) \\
& +f_{\Gamma} \dot{\tilde{p}}^{*}(x, y) q(y) \mathrm{d} \Gamma(y)+f_{\Gamma} \tilde{p}^{*}(x, y) q(y) \mathrm{d} \Gamma(y),
\end{aligned}
$$

where the upper $\operatorname{dot}(\dot{)}$ denotes differentiation with respect to the design variable, and $\dot{p}^{*}(x, y)$ can be related to the sensitivities of the coordinate as

$$
\dot{p}^{*}(x, y)=-\frac{e^{i k r}}{4 \pi r^{2}}(1-i k r) \frac{\partial r}{\partial y_{i}}\left(\dot{y}_{i}-\dot{x}_{i}\right),
$$

and $\dot{q}^{*}(x, y), \dot{\tilde{p}}^{*}(x, y)$ and $\dot{\tilde{p}}^{*}(x, y)$ can also be expressed in the form as $\dot{p}^{*}(x, y)$.

Both eqns (13) and (14) can be used for acoustic boundary sensitivity analysis. But for an exterior acoustic problem, similar to eqns (6) and (7), they also have a different set of fictitious eigenfrequencies, and the linear combination of them can also provide unique solutions for all frequencies.

Discretizing the linearly composite formulation of eqns (13) and (14), and collecting the equations for all the collocation points and writing them in matrix forms result in the following form of linear algebraic equations

$$
[H]\{\dot{p}\}=[G]\{\dot{q}\}-[h]\{p\}+[g]\{q\},
$$

where $[H]$ and $[G]$ are the same matrices as those in eqn (10), but $[h]$ and $[g]$ are the newly derived matrices that must be computed here. Also, the unknown terms of $\{p\}$ and $\{q\}$ in eqn (16) can be obtained by solving eqn (11). 
$\dot{p}$ (or $\dot{q}$ ) is also known on the boundary where $p$ (or $q$ ) is prescribed. Therefore, eqn (16) can be rearranged by using the boundary conditions for $\dot{p}$ and $\dot{q}$ as follows

$$
[A]\{\dot{x}\}=[B]\{\dot{y}\}-[h]\{p\}+[g]\{q\},
$$

where $[A]$ and $[B]$ are the same matrices as those in eqn (11), $\{\dot{x}\}$ and $\{\dot{y}\}$ are the unknown and known vectors, respectively. Equation (17) can now be solved and all the boundary sensitivity values are then known. Once this has been done, it is possible to calculate $\dot{p}(x)$ at any point within the problem domain by using eqn (12).

The strongly-singular or hypersingular boundary integrals can be found in eqns (6), (7), (13) and (14). In order to calculate them accurately, eqns (6), (7), (13) and (14) are often regularized by using the fundamental solution of the Laplace's equation. But the regularized equations are not efficient to use in the FMBEM, because multipole expansion formulas and other translation formulas have to be implemented not only for the fundamental solution and its derivatives of the Helmholtz's equation but also for those of the Laplace's equation. However, when the constant triangular element is used to discretize the boundary, these strongly singular and hypersingular integrals can be evaluated in the sense of finite parts without any difficulty, hence FMBEM can be applied efficiently. The details of evaluating such integrals for constant triangular elements can be found in $[5,6]$.

\subsection{FMBEM formulations}

In this section, the FMBEM is employed to solve the problems, and an iterative solver, the generalized minimal residual method (GMRES), is used in the solution process because the system of linear equations (11) and (17) is not formed explicitly. Several expansion and translation formulas needed in the procedure of the FMBEM have been well presented in [7].

The fundamental solution (8) can be expanded into the following series around an expansion point $O$ near $y$

$$
p^{*}(x, y)=\frac{i k}{4 \pi} \sum_{n=0}^{\infty} \sum_{m=-n}^{n}(2 n+1) \bar{I}_{n}^{m}(k, \overrightarrow{O y}) O_{n}^{m}(k, \overrightarrow{O x}), \quad|\overrightarrow{O x}|>|\overrightarrow{O y}|,
$$

where

$$
\begin{aligned}
I_{n}^{m}(k, \overrightarrow{O y}) & =j_{n}(k|\overrightarrow{O y}|) Y_{n}^{m}(\widehat{O y}), \\
O_{n}^{m}(k, \overrightarrow{O x}) & =h_{n}^{(1)}(k|\overrightarrow{O x}|) Y_{n}^{m}(\widehat{O x})
\end{aligned}
$$

and $\bar{I}_{n}^{m}$ is the complex conjugate of $I_{n}^{m}, j_{n}$ and $h_{n}^{(1)}$ are the $n$-th order spherical Bessel function of the first and third kind, and $Y_{n}^{m}$ is the spherical harmonics. 


\subsubsection{FMBEM formulations for acoustic state analysis}

In the FMBEM procedure, we calculate first the following multipole moments for the boundary element $j$ far away from the collocation point $x\left(|\overrightarrow{O x}|>\left|\overrightarrow{O y_{j}}\right|\right)$.

$$
M_{n}^{m}\left(k, \overrightarrow{O y_{j}}\right)= \begin{cases}\int_{\Gamma_{j}} \frac{\partial \bar{I}_{n}^{m}(k, \overrightarrow{O y})}{\partial n(y)} p(y) \mathrm{d} \Gamma(y) & \text { for } H^{i j} p^{j} \\ \int_{\Gamma_{j}} \bar{I}_{n}^{m}(k, \overrightarrow{O y}) q(y) \mathrm{d} \Gamma(y) & \text { for } G^{i j} q^{j} .\end{cases}
$$

Multipole moments of a group of $\ell$ elements that are close to $O$ (more precisely, all elements in the same LEAF-cell whose center is point $O$ ) can be added up in order to form $M_{n}^{m}(k, O)$ that is the multipole moments centered at $O$.

$$
M_{n}^{m}(k, O)=\sum_{j=1}^{\ell} M_{n}^{m}\left(k, \overrightarrow{O y_{j}}\right)
$$

The center of the multipole moment can be shifted from $O$ to $O^{\prime}$ according to the following Moments to Moments (M2M) translation formulation, if $\left|\overrightarrow{O^{\prime} x}\right|>\left|\overrightarrow{O^{\prime} y}\right|$.

$$
\begin{aligned}
M_{n}^{m}\left(k, O^{\prime}\right)= & \sum_{n^{\prime}=0}^{\infty} \sum_{m^{\prime}=-n^{\prime}}^{n^{\prime}} \sum_{\substack{l=\left|n-n^{\prime}\right| \\
n^{\prime}+n-l: \text { even }}}^{n+n^{\prime}}\left(2 n^{\prime}+1\right)(-1)^{m^{\prime}} W_{n, n^{\prime}, m, m^{\prime}, l} \\
& \times I_{l}^{-m-m^{\prime}}\left(k, \overrightarrow{O^{\prime} O}\right) M_{n^{\prime}}^{-m^{\prime}}(k, O),
\end{aligned}
$$

where $W_{n, n^{\prime}, m, m^{\prime}, l}$ is given by

$$
W_{n, n^{\prime}, m, m^{\prime}, l}=(2 l+1) i^{n^{\prime}-n+l}\left(\begin{array}{ccc}
n & n^{\prime} & l \\
0 & 0 & 0
\end{array}\right)\left(\begin{array}{ccc}
n & n^{\prime} & l \\
m & m^{\prime} & -m-m^{\prime}
\end{array}\right),
$$

and $(\cdot . \quad . \quad$.$) stands for the Wigner 3 \mathrm{j}$ symbol [8].

The Moments to Local expansion (M2L) translation can be expressed as

$$
\begin{aligned}
L_{n}^{m}\left(k, x_{0}\right)= & \sum_{n^{\prime}=0}^{\infty} \sum_{m^{\prime}=-n^{\prime}}^{n^{\prime}} \sum_{\substack{l=\left|n-n^{\prime}\right| \\
n^{\prime}+n-l: \text { even }}}^{n+n^{\prime}}\left(2 n^{\prime}+1\right)(-1)^{m+m^{\prime}} W_{n^{\prime}, n, m^{\prime}, m, l} \\
& \times O_{l}^{m+m^{\prime}}\left(k, \overrightarrow{O^{\prime} x_{0}}\right) M_{n^{\prime}}^{m^{\prime}}\left(k, O^{\prime}\right) .
\end{aligned}
$$


The local expansion center can be shifted from $x_{0}$ to $x_{1}$ according to the following Local expansion to Local expansion (L2L) translation formulation,

$$
\begin{aligned}
L_{n}^{m}\left(k, x_{1}\right)= & \sum_{n^{\prime}=0}^{\infty} \sum_{m^{\prime}=-n^{\prime}}^{n^{\prime}} \sum_{\substack{l=\left|n-n^{\prime}\right| \\
n^{\prime}+n-l: \text { even }}}^{n+n^{\prime}}\left(2 n^{\prime}+1\right)(-1)^{m} W_{n^{\prime}, n, m^{\prime},-m, l} \\
& \times I_{l}^{m-m^{\prime}}\left(k, \overrightarrow{x_{0} x_{1}}\right) L_{n^{\prime}}^{m^{\prime}}\left(k, x_{0}\right) .
\end{aligned}
$$

Finally, $\sum_{j=1}^{\ell} H^{i j} p^{j}$ or $\sum_{j=1}^{\ell} G^{i j} q^{j}$ can be expressed in terms of the local expansions using local expansion coefficients obtained from eqns (25) or (26) as

$$
\begin{aligned}
\sum_{j=1}^{\ell} H^{i j} p^{j} \text { or } \sum_{j=1}^{\ell} G^{i j} q^{j}= & \frac{i k}{4 \pi} \sum_{n=0}^{\infty} \sum_{m=-n}^{n}(2 n+1) L_{n}^{m}\left(k, x_{1}\right) \\
& \times\left(\bar{I}_{n}^{m}\left(k, \overrightarrow{x_{1} x}\right)+\alpha \frac{\partial \bar{I}_{n}^{m}\left(k, \overrightarrow{x_{1} x}\right)}{\partial n(x)}\right) .
\end{aligned}
$$

For elements that are close to the collocation point $x$, the conventional method in Section 2.1.1 should be used.

\subsubsection{FMBEM formulations for acoustic sensitivity analysis}

The computation of $\sum_{j=1}^{\ell} H^{i j} \dot{p}^{j}$ or $\sum_{j=1}^{\ell} G^{i j} \dot{q}^{j}$ by using the FMBEM is similar to that of $\sum_{j=1}^{\ell} H^{i j} p^{j}$ or $\sum_{j=1}^{\ell} G^{i j} q^{j}$ in the above section, so only the computation of $\sum_{j=1}^{\ell}\left(g^{i j} q^{j}-h^{i j} p^{j}\right)$ is discussed next.

It is easy to find that the computation of $\sum_{j=1}^{\ell}\left(g^{i j} q^{j}-h^{i j} p^{j}\right)$ should be done in two steps, the multipole moments for these two steps are defined, as follows:

$$
{ }^{1} M_{n}^{m}\left(k, \overrightarrow{O y_{j}}\right)=\int_{\Gamma_{j}} \bar{I}_{n}^{m}(k, \overrightarrow{O y}) q(y) \mathrm{d} \Gamma(y)-\int_{\Gamma_{j}} \frac{\partial \bar{I}_{n}^{m}(k, \overrightarrow{O y})}{\partial n(y)} p(y) \mathrm{d} \Gamma(y),
$$

and

$$
\begin{aligned}
& { }^{2} M_{n}^{m}\left(k, \overrightarrow{O y_{j}}\right)=\int_{\Gamma_{j}}\left(\frac{\partial \bar{I}_{n}^{m}(k, \overrightarrow{O y})}{\partial y_{i}} \dot{y}_{i} q^{j}\right) \mathrm{d} \Gamma(y)+\int_{\Gamma_{j}} \bar{I}_{n}^{m}(k, \overrightarrow{O y}) q^{j} \mathrm{~d} \Gamma(y) \\
& -\int_{\Gamma_{j}}\left(\frac{\partial^{2} \bar{I}_{n}^{m}(k, \overrightarrow{O y})}{\partial y_{i} \partial y_{j}} n_{i}(y) \dot{y}_{j} p^{j}\right) \mathrm{d} \Gamma(y)-\int_{\Gamma_{j}}\left(\frac{\partial \bar{I}_{n}^{m}(k, \overrightarrow{O y})}{\partial y_{i}} \dot{n}_{i}(y) p^{j}\right) \mathrm{d} \Gamma(y) \\
& -\int_{\Gamma_{j}}\left(\frac{\partial \bar{I}_{n}^{m}(k, \overrightarrow{O y})}{\partial y_{i}} n_{i}(y) p^{j}\right) \mathrm{d} \Gamma(y) .
\end{aligned}
$$

The M2M, M2L, L2L translation formulations for the two steps are the same as eqns (23), (25) and (26). Finally, $\sum_{j=1}^{\ell}\left(g^{i j} q^{j}-h^{i j} p^{j}\right)$ can be expressed in terms 
of the local expansions as

$$
\begin{aligned}
& \sum_{j=1}^{\ell}\left(g^{i j} q^{j}-h^{i j} p^{j}\right)=\frac{i k}{4 \pi} \sum_{n=0}^{\infty} \sum_{m=-n}^{n}(2 n+1)^{1} L_{n}^{m}\left(k, x_{1}\right)\left(\frac{\partial \bar{I}_{n}^{m}\left(k, \overrightarrow{x_{1} x}\right)}{\partial x_{i}} \dot{x}_{i}\right. \\
& \left.+\alpha \frac{\partial^{2} \bar{I}_{n}^{m}\left(k, \overrightarrow{x_{1} x}\right)}{\partial x_{i} \partial x_{j}} n_{i}(x) \dot{x}_{j}+\alpha \frac{\partial \bar{I}_{n}^{m}\left(k, \overrightarrow{x_{1} x}\right)}{\partial x_{i}} \dot{n}_{i}(x)\right) \\
& +\frac{i k}{4 \pi} \sum_{n=0}^{\infty} \sum_{m=-n}^{n}(2 n+1)^{2} L_{n}^{m}\left(k, x_{1}\right)\left(\bar{I}_{n}^{m}\left(k, \overrightarrow{x_{1} x}\right)+\alpha \frac{\partial \bar{I}_{n}^{m}\left(k, \overrightarrow{x_{1} x}\right)}{\partial n(x)}\right)
\end{aligned}
$$

For elements that are close to the collocation point $x$, the integrals are evaluated with the conventional method stated in Section 2.1.2.

\section{Numerical examples}

A pulsating sphere example that has the analytical solution is chosen to verify the validity of the method. The radius $a$ of the sphere is designated as a shape design variable. The partial derivative of the sound pressure at a field point with respect to this radius is calculated and compared with the analytical solution.

For a sphere vibrating with the velocity $v_{n}$ in the radial direction, the sensitivity of the pressure with respect to a change of radius becomes

$$
\frac{\partial p(r)}{\partial a}=\frac{\rho c k a}{r}\left\{\frac{\partial v_{n}}{\partial a} \frac{i a}{1+i k a}+v_{n}\left[\frac{2 i-k a}{1+i k a}+\frac{k a}{(1+i k a)^{2}}\right]\right\} e^{-i k(r-a)},
$$

where $r$ is a distance from the center of the sphere to the field point of interest.

In the numerical analysis, we assumed as $a=1.0 \mathrm{~m}, v_{n}=1.0 \mathrm{~m} / \mathrm{s}$, and sensitivities are calculated for a field point with $r=6.0 \mathrm{~m}$. The acoustic medium is assumed to be air with a density of $\rho=1.2 \mathrm{~kg} / \mathrm{m}^{3}$ and a sound velocity of $c=340 \mathrm{~m} / \mathrm{s}$. The values of $\dot{x}_{i}$ used for eqns (29) and (30) were given, for simplicity, as the finite difference approximations calculated from the radius augmented by $1 \%$.

Figures 1 and 2 show the result for a 1078 constant triangular element model with the wave numbers varying from 0.5 to 5.0. The solutions of real and imaginary part of the sound pressure sensitivities are shown. It can be seen that the CBIE or NDBIE solution follows the analytical solution closely except in the vicinity of the fictitious eigenfrequencies. But the solution based on BurtonMiller's Method is very accurate over the entire range of the wave number.

Figures 3 and 4 show comparisons of relative errors and CPU times between FMBEM, CBEM-LU and CBEM-GMRES. In all the computations, the maximum number of elements in a leaf is set to 100 , and the truncation number of multipole and local expansion terms is set to10. The GMRES solver is to stop iterations when the residual becomes smaller than the tolerance $10^{-3}$, and $k$ is set to 0.5. All computations were done on a $\mathrm{PC}$ with an Intel $3.0 \mathrm{GHz}$ processor and 2GB memory. Figure 3 shows that the percentage relative errors of the FMBEM, 


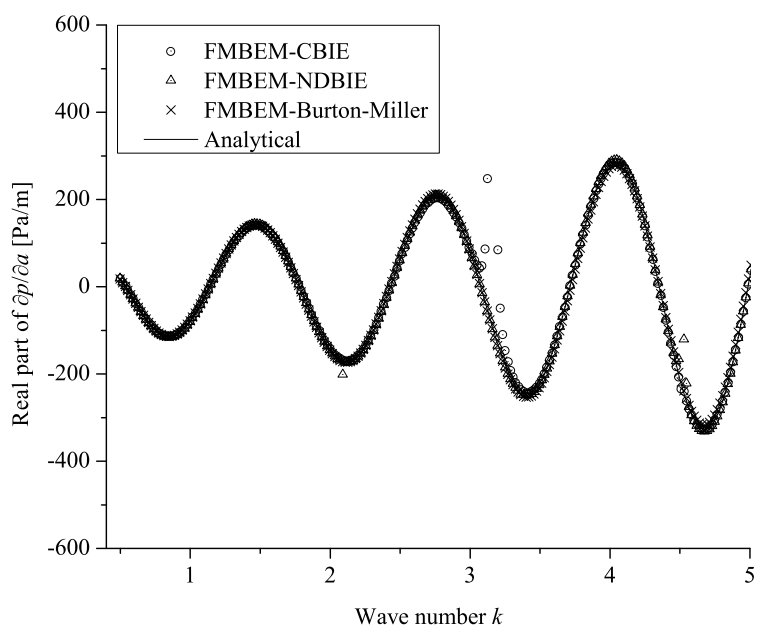

Figure 1: Results for real part of $\partial p(r) / \partial a$.

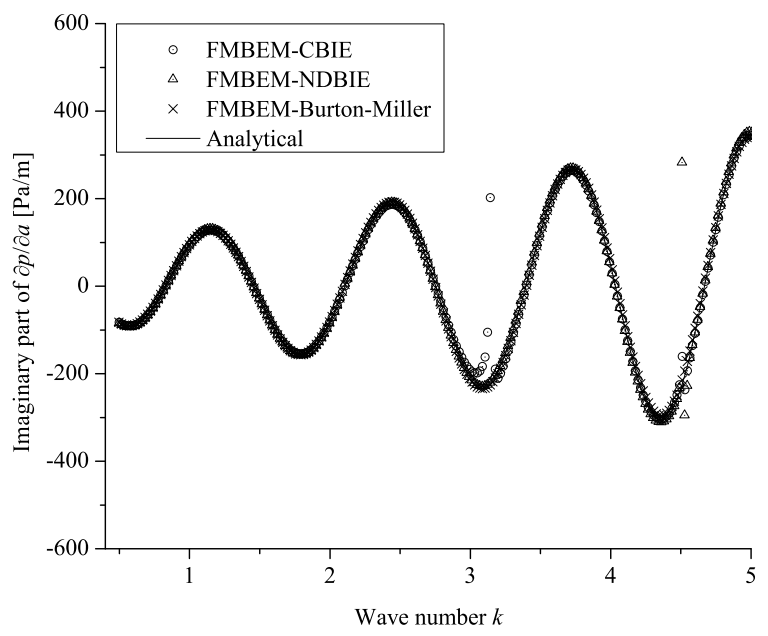

Figure 2: Results for imaginary part of $\partial p(r) / \partial a$.

CBEM-LU and CBEM-GMRES are very close to each other, and all errors decrease very fast as the number of elements increases. It is found from Figure 4 that the FMBEM is faster than the CBEM-LU for models with more than 4000 elements, and the FMBEM is also faster than the CBEM-GMRES for models with more than 7000 elements. 


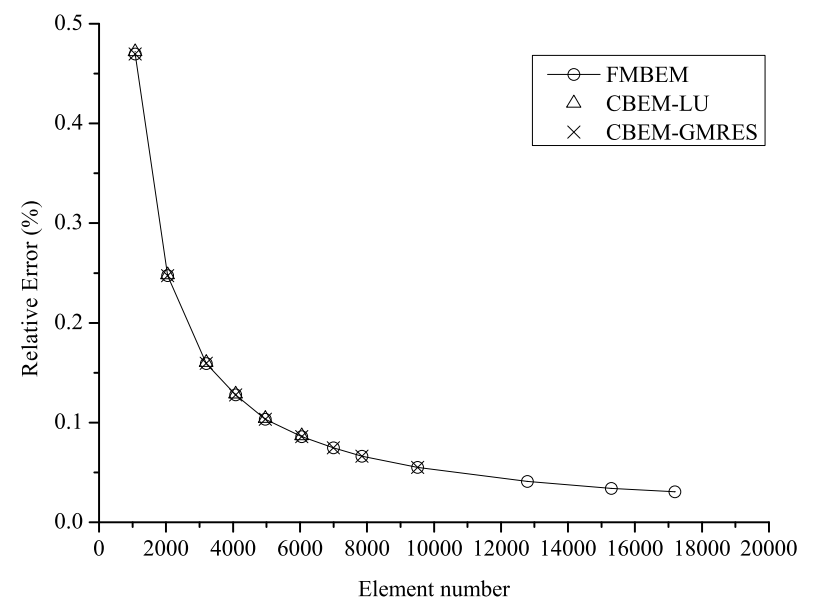

Figure 3: Comparison of relative error.

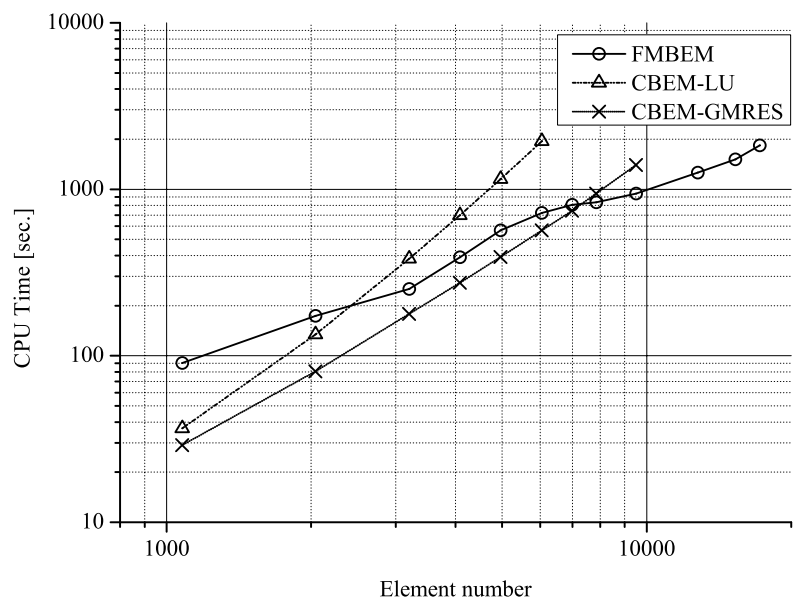

Figure 4: Comparison of CPU time.

\section{Conclusions}

A FMBEM approach has been presented in this paper for 3-D acoustic design sensitivity analysis using the boundary integral equation of shape design sensitivity coefficients of the sound pressure and particle velocity. Since the so-called fictitious eigenfrequencies are also observed for the sensitivity analysis for exterior acoustic fields, the Burton-Miller approach, which uses a linear combination of CBIE and NDBIE for the sensitivity coefficients, is employed to overcome the problem. 
The multipole moments and M2M, M2L, L2L translation formulas have been presented for the derived sensitivity boundary integral equations. Some numerical results for a pulsating sphere has been shown to demonstrate the validity and computation efficiency of the present approach.

\section{References}

[1] Burton, A.J. \& Miller, G.F., The application of integral equation methods to the numerical solution of some exterior boundary-value problems. Proc. Roy. Soc. Lond., A. 323, pp. 201-210, 1971.

[2] Rokhlin, V., Rapid solution of integral equations of classical potential theory. Journal of Computational Physics, 60(2), pp. 187-207, 1985.

[3] Nishimura, N., Fast multipole accelerated boundary integral equation methods. ASME, 55(4), pp. 299-324, 2002.

[4] Kress, R., Minimizing the condition number of bounday integral operators in acoustic and electromagnetic scattering. Q. J. Mech Appl Math, 38, pp. 323243, 1985.

[5] Matsumoto, T., Zheng, C.J., Harada, S. \& Takahashi, T., Explicit evaluation of hypersingular boundary integral equation of 3-D Helmholtz equation for constant triangular element discretization. Submitted to JSME Technical Journal.

[6] Zheng, C.J., Matsumoto, T., Takahashi, T. \& Chen, H.B., Boundary element shape design sensitivity formulation of 3D acoustic problems based on direct differentiation of strongly-singular and hypersingular boundary integral equations. Submitted to JSME.

[7] Yoshida, K., PhD Thesis, Kyoto University, Japan, 2001.

[8] Abramowitz, M. \& Stegun, I.A., Handbook of Mathematical Functions with Formulas, Dover: New York, pp. 1006-1010, 1972.

[9] Matsumoto, T., Tanaka, M. \& Yamada, Y., Design Sensitivity Analysis of Steady-State Acoustic Problems using Boundary Integral Equation Formulation. JSME International Journal, Series C, 38(1), pp. 9-16, 1995.

[10] Arai, Y., Tanaka, M. \& Matsumoto, T., A New Design Sensitivity Analysis of Acoustic Problems Based on Bem Avoiding Fictitious Eigenfrequency Issure. (in Japanese) JSME, Series C, 73(729), pp. 120-127, 2007.

[11] Shen, L. \& Liu, Y.J., An adaptive fast multipole boundary element method for three-dimensional acoustic wave problems based on the Burton-Miller formulation, Comput Mech, 40, pp. 461-472, 2007. 